\title{
Phase transition in Gold nuclei at 10.6A GeV
}

\author{
P. L. Jain, G. Singh and R. Gonsalves \\ High Energy Experimental Laboratory \\ Department of Physics \\ State University of New York at Buffalo Buffalo, New York 14260
}

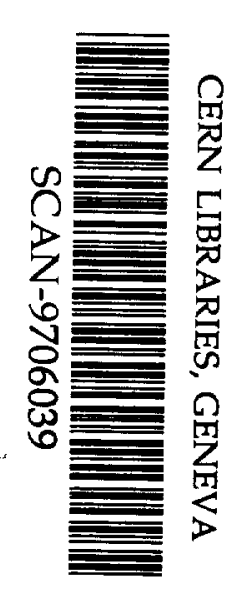

May 5, 1997

\begin{abstract}
The charge $(Z)$ distribution of multifragment decays of ${ }^{197} \mathrm{Au}$ projectile at $10.6 \mathrm{~A} \mathrm{GeV}$ in nuclear emulsion is fitted with a power-law. The moments of nuclear charged fragment distribution provide strong evidence that nuclear matter possesses critical point observables. The observed values of the critical exponents $\gamma, \beta$ and $\tau$ are very close to those for a liquid-gas system.
\end{abstract}

\section{PACS Numbers: $25.75 .+\mathrm{r}, 25.70 \mathrm{Pq}, 0.5 .70 \mathrm{Jk}$}

At present there is a great deal of interest in the extent to which the phenomenon of multifragmentation, which has been studied extensively in theoretical calculations, is related to possible phase transitions of nuclear matter [1]. Finn et al. [2] first observed the power-law behavior in the fragment charge distribution and their work suggested that perhaps nuclear fragmentation was a critical phenomenon occurring in a small system. Since that time, a lot of experimental work has been done on this subject. Further progress in the field of multifragmentation [3,4] has indicated that this powerlaw behavior alone is only suggestive but not conclusive of critical behavior. Critical behavior is associated with a finite number of critical indices which characterize the sharp change in the properties of a substance. In heavy and finite size systems like ${ }^{197} \mathrm{Au}$ nuclei at $10.6 \mathrm{~A} \mathrm{GeV}$, it is still possible to study the vestiges of a phase transition and the critical exponents through their nuclear fragmentation process, and that is the subject of this letter. Previously [5], we have studied the liquid-gas co-existence phase in =emulsion interactions at $160 \mathrm{~A} \mathrm{GeV} \mathrm{[5].} \mathrm{We}$ present here the exclusive data for the determination of critical exponents associated with multifragmentation of a ${ }^{197} \mathrm{Au}$ beam at $10.6 \mathrm{~A} \mathrm{GeV}$ for the first time and compare these values with those at low energy $(\approx 1 \mathrm{~A} \mathrm{GeV}){ }^{197} \mathrm{Au}$ beam [4], with the ${ }^{207} \mathrm{~Pb}$ beam at $160 \mathrm{~A} \mathrm{GeV} \mathrm{[5]} \mathrm{and} \mathrm{with} \mathrm{liquid-gas} \mathrm{systems}$ [1]. Earlier in the projectile fragmentation work of ${ }^{197} \mathrm{Au}$ beam at $10.6 \mathrm{~A} \mathrm{GeV} \mathrm{[6]}$, it was shown that the energy deposition in the projectile fragments (PFs) does not vary much from that at low energy $\approx 1 \mathrm{~A}$ $\mathrm{GeV}$. From the observations of the PFs it is important to extract the values of the critical exponents of ${ }^{197} \mathrm{Au}$ beam at $10.6 \mathrm{~A} \mathrm{GeV}$ and to check the validity of this technique [4] for future work. It may be pointed out that the authors of Ref. [4] have raised some very basic questions regarding the thermal equilibrium of the system and whether the multiplicity is proportional to temperature. These questions were not answered previously in Ref. [4].

We first answer the questions raised above by using our previous [7] and the present data samples on intermediate-mass-fragments (IMFs). The correlation between $\left\langle N_{I M F}\right\rangle$ multiplicity and $N_{p}$ is almost independent of the target nucleus, where $N_{p}=Z_{\text {beam }}-Z_{\text {boundi }}, Z_{\text {bound }}=\sum_{Z_{i}>1} Z_{i}$ and $Z_{\text {heam }}$ is the beam charge. $Z_{\text {hound }}$ is complement of $N_{p}$, the multiplicity of emitted hydrogen nuclei from the projectile nucleus. The quantity $Z_{\text {bound }}$ also discloses indirectly the amount of nuclear matter contained in all fragments. The universal behavior between $\left\langle N_{I M F}>\right.$ vs $N_{p}$ suggests that large degree of equilibrium is obtained prior to its decay and that $N_{p}$ is sensitive to energy deposition, and thus to the excitation energy of the spectator matter. Thermal equilibrium is further depicted in Fig. 1(b) of Ref. [7], where the universal correlation be tween the distribution of $\left\langle N_{I M F}\right\rangle \mid\left\langle N_{\max }\right\rangle$ and $N_{p} / Z_{\text {beam }}$ is shown for four different beams at $\approx 1 \mathrm{~A} \mathrm{GeV}$ along with ${ }^{197} \mathrm{Au}$ at $10.6 \mathrm{~A} \mathrm{GeV} \mathrm{[7].} \mathrm{Here}$ $\left\langle N_{\max }\right\rangle$ is the average multiplicity of maximum number of IMFs in a given data set. All four beams

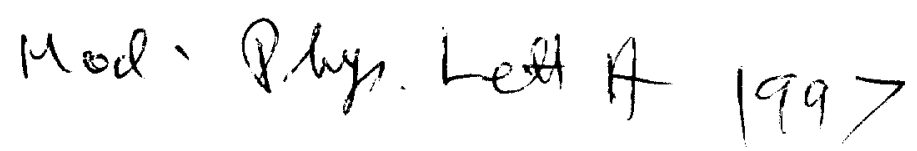


have the general characteristics of rise and fall [8] passing through their maxima [7]. The peak in al cases is approximately at the midcenter. The underlying mechanism responsible for the magnitude of these IMFs is strongly correlated with the excitation energy of the projectile through the number of $N_{p}$ or $Z_{\text {bound }}$. It is quite fair to state that the decaying spectator nuclei are thermally equilibrized.

By following along the primary track of ${ }^{197} \mathrm{Au}$ ions at 10.6A GeV from BNL Experiment No. 875, we observed 1700 nuclear interactions. For the present analysis, we selected 1220 events having at least one projectile fragment of charge $Z \geq 2$ in emulsion which has the best spatial resolution. In each event. the charges of all the PFs were determined as discussed in Ref. [9]. The charges of all the PFs were determined by a combination of different methods: grain, gap and $\delta$-ray density and track widths (as discussed previously $[6,7,9]$ ) along with their charge resolution. The accuracy in the angular measurements in emulsion is better than $0.1 \mathrm{mrad}$ for angles $\theta \leq 1 \mathrm{mrad}$. The angles of PFs were measure very accurately. The $1 \mathrm{MFs}$ for ${ }^{197} \mathrm{Au}$ at $10.6 \mathrm{~A} \mathrm{GeV}$ were considered between $2 \leq Z \leq 23$. First we look at the growth process of fragments for the charge distribution $\ln \left(N_{Z}\right)$ as a function of $\ln Z$ for an inclusive data, on an event-by-event basis, where $N_{Z}$ stands for the total number of projectile fragments corresponding to a given charge $Z$. This distribution is presented in Fig. 1(a) for the ${ }^{197} \mathrm{Au}$ data at $10.6 \mathrm{~A} \mathrm{GeV}$. If we consider charges $2 \leq Z \leq 23$, the solid line follows an inverse power-law $\left(Z^{-\tau}\right)$ with an exponent value of $\tau=-2.153 \pm 0.165$. For $3 \leq Z \leq 11$, Fig. 1(b) exhibits the normalized probability distributions of PFs. They were fitted with an exponential function $\left(e^{-\alpha Z}\right)$, where the fitted value of $\alpha=0.28 \pm 0.04$ with $\chi^{2}=0.12$ and is exhibited with the dotted line in the figure. The errors shown here are all statistical unless otherwise stated. We further look at the charge distribution of multifragments between $3 \leq Z \leq 11$ produced in interactions, accompanied with two categories of alphas, with their transverse momenta $P_{t}<200$ $\mathrm{MeV} / \mathrm{c}$ and $P_{t} \geq 200 \mathrm{MeV} / \mathrm{c}$. In Fig. 1(c) is shown the normalized fragment probability from both types of alphas. Both the distributions are practically equal and can be fitted with an exponential function $\left(e^{-\alpha Z}\right)$, where $\alpha=0.76 \pm 0.22$ with $\chi^{2}=1.8$. This indicates that the thermalized excitation energies are quite close to one and other in both types of interactions. In stead of tagging the events with two types of alphas, we now take the whole data sam- ple and plot the multiplicity distributions of PFs of charges $3 \leq Z \leq 11$ with (a) $N_{p} \leq 28$ for peripheral and (b) $28<N_{p} \leq 60$ for mid-central events. Once again both the distributions have approximately the same slope $\alpha=0.28 \pm 0.04$ with $\chi^{2}=0.23$, which is exhibited in Fig. 1(d). Figs. 1(c) and 1(d) answer the first part of the question raised earlier.

In order to check further on the thermal nature of the PFs produced in ${ }^{197} \mathrm{Au}$ beam at $10.6 \mathrm{~A} \mathrm{GeV}$, we need to know the excitation energy of the event or at least a quantity proportional to it. Here we assume the transverse energy $\left(E_{t}=\Sigma E_{i} \sin \theta_{i}\right.$, where $E_{i}$ and $\theta_{i}$ are the kinetic energy and the laboratory angle of each fragment, respectively) is proportional to the excitation energy $E^{*}$ and $E^{*}$ depends upon the value of $Z_{\text {bound }}$. It is independent of the specific target and of the bombarding energy [10]. We used a subsample of about 400 events [9] which have at least four PFs of charge $3 \leq Z \leq 11$. Fig. 2(a) depicts the log of such fragment emissions when plotted against $1 / E_{t}^{1 / 2}$ (Arrhenius plot). The decay probability of N PFs is fitted with an exponential function $e^{-\alpha / E_{t}{ }^{1 / 2}}$. It gives a straight line. The linear dependence is strongly suggestive that there was a large degree of thermal equilibrium in the decay system of the excited spectators (PFs) at different impact parameters over the measured $E_{t}$ range. Assuming a degenerate Fermi gas, nuclear temperature and excitation energy are related as $T \propto E_{t}^{1 / 2}$, where $T$ is the temperature of the emitting system. Fig. 2(b) exhibits the correlation of $E_{t}^{1 / 2}$ vs $Z_{\text {bound }}$ which is proportional to the impact parameter [9] and gives a linear relation. The linearity is strongly suggestive of thermal equilibrium in the decaying system of the excited spectator (PFs) at different impact parameters over the measured $E_{\mathrm{t}}$ range. This answers the second part of the question raised earlier.

In the present discussion, we follow Campi's [3] suggestion that the moments of the fragment distribution should exhibit features characteristic of critical phenomena if the fragments were produced in a system near its critical point. In percolation theory, according to Stauffer [1], the moments of the cluster distribution contain the signals for critical behavior. Fragment distributions are characterized by the $k$ moments of the distribution [3]: $S_{k}=\sum_{Z} Z^{k} n_{Z}$. Here, $n_{Z}$ is the number of PFs of charge $Z$ in an event. The sum runs over all the fragments of $Z \geq 1$ excluding the heaviest one produced in an event, multiplicity $n$ is the number of charged frag- 
ments - $Z_{\max }$. For a finite system, one finds the region in probability where the power-law behavior of $S_{k}=\sum_{Z} Z^{k} n_{Z}$ holds. In Fig. 2(c) is shown the behavior of the second moments $<\ln S_{2}>$ vs $n$ for ${ }^{197} \mathrm{Au}$ beam at $10.6 \mathrm{~A} \mathrm{GeV} . S_{2}$ value reaches a maximum which is a manifestation of a phase transition for an intermediate value of multiplicity at $n_{c} \approx 22$. Near the critical point $\left(n_{c}\right), S_{2}$ shows fluctuations and then it decreases very slowly. These fluctuations can be interpreted as a signal that the event spectrum contains 'liquid', 'liquid-gas' and 'gas' phases corresponding to regions below, at and beyond $n_{c}$, respectively. If we plot $\left\langle\ln S_{2}>\right.$ vs $N_{p}$, the number of projectile (spectator) protons, we get approximately the same curve as shown in Fig. 2(c). This curve further explains the effect of the impact parameter through the number of $N_{p}$ in the interactions which are quite distinctive from the cascade remnant of the target in emulsion at relativistic energies. A monotonic relationship between the number $N_{p}$ or $Z_{\text {hound }}$ and the impact parameter (b) has been shown previously for ${ }^{197} \mathrm{Au}$ beam [9]. The broad maximum in place of a singularity is due to the finite size of the system and for which we have omitted $Z_{\max }$ from the liquid side $[1,3]$ and in all of the following calculations.

In the critical region, we are to find in an independent manner three critical exponents $\gamma, \beta$ and $\tau$, and determine whether these exponents obey the well known sumrules. Critical exponents for a large system are defined as:

$$
\begin{gathered}
\text { (a) } S_{2} \approx\left|n-n_{c}\right|^{-\gamma}, \quad \text { (b) } Z_{\max } \approx\left|n-n_{c}\right|^{\beta} \\
\text { and }(c) N_{Z} \approx Z^{-\tau}
\end{gathered}
$$

where $\left(n-n_{c}\right)$ is the distance from critical multiplicity. Eq. (1) can be applied to thermal as well as to the percolation models. In most statistical and percolation models, there are two independent exponents, and the three exponents $(\gamma, \beta$ and $\tau$ ) are not independent, but obey the sumrule: $\tau=2+\beta /(\beta+\gamma)$. For a finite system the second moment $S_{2}$ will have a maximum value (instead of infinity), and $\tau$ goes through a minimum at the critical point. Thus, $\tau$ and $S_{2}$ can be used to identify the critical point in a finite system within a certain nuclear density range. Nuclear density varies from event to event, as does their temperature. When the nuclear density is higher than a minimum value, a critical point will also be reached at a certain higher temperature. Hence it is justified to use the inclusive data from a thermalized nuclear system to determine the critical exponents at the point where either maximum in $S_{2}$ or a minimum in $\tau$ is achieved.

In nuclear collisions, matter is compressed, heated up and one can talk of a freeze-out density $(\rho)$ and temperature $(T)$ at the time of dissociation. Both $T$ and $\rho$ are, however, not directly measured. But, we have shown earlier how to get information about $\mathrm{T}$. The most commonly measurable quantities are the multiplicity of charged fragments $(n), S_{2}$ and $Z_{\max }$. For a given $\mathrm{n}$ both $S_{2}$ and $Z_{\max }$ can vary from one event to another, and hence one generally plots the average for a given $n$. In order to measure energy density $\epsilon_{0}$, within the thermally equilibrated region, we used nuclear interactions with $N_{p} \leq 60$ and measured the multiplicities and the angles of all the shower particles $\left(N_{s}\right)$ produced in the interactions. We calculated the average particle density per unit of pseudorapidity $\langle d n / d \eta\rangle$ in range $0.5 \leq \eta \leq 3.7$ for shower $N_{s}$ particles. We applied the Bjorken formula for $\epsilon_{0}$, where $\left\langle p_{t}\right\rangle=350$ $\mathrm{MeV} / \mathrm{c}, A=197$ and $\tau_{0} \approx 1$ and in Fig. $2(d)$ is shown the distribution of $\epsilon_{0}$ vs $N_{s}$, where energy density increases with multiplicity.

After establishing the thermal nature of PFs, we can then discuss the determination of the exponent $\gamma$ in $S_{2} \approx\left(n-n_{c}\right)^{-\gamma}$. We find a region where the power-law behavior holds. The value of $\gamma$ depends on the region of $\left|n-n_{c}\right|$ used since finite size distortions dominate as $\left|n-n_{c}\right| \rightarrow 0$, and the signature of critical behavior vanishes for a large $\left|n-n_{c}\right|$ i.e. in the mean field regime. We choose a trial value of $n_{c}$ such that for the liquid side of $n_{c}$, the plot of $\ln \left(S_{2}\right)$ versus $\ln \left|\left(n-n_{c}\right)\right|$ gives the same value of $\gamma$ as on the gas side so that $\left|\gamma_{g}-\gamma_{l}\right| \approx$ minimum. Using a trial and error method, we find that $n_{c}=22$ satisfies our requirement and this is shown in Fig. 3(a), where the value of $\left|\gamma_{g}-\gamma_{t}\right|$ is plotted against $n_{c}$. For different $n_{c}$ values of $n_{c}<22$, or $>22$, the difference in the values of $\left|\gamma_{g}-\gamma_{l}\right|$ increases as one departs from $n_{c}=22$. The distribution of $<\ln S_{2}>$ vs $\ln \left|n-n_{c}\right|$ is shown in Fig. 3(b) for the liquid side $\left(n<n_{c}\right)$ with $\gamma_{t}=1.118 \pm 0.153$ and for the gas side $\left(n>n_{c}\right)$ in Fig. $3(\mathrm{c})$ with $\gamma_{g}=1.228 \pm 0.121$. The $\gamma$ values are the same within the statistical errors and the difference between $\gamma_{t}$ and $\gamma_{g}$ is $<10 \%$. The average value of $\gamma$ is given in Table I. The straight lines are the best fits to the data points. But these values differ slightly from that predicted by percolation theory [1]. We use the same value of $n_{c}=22$ to get the value of $\beta$ for the distribution of $Z_{\max } \approx\left|n-n_{c}\right|^{\beta}$ on the liquid side from Eq. (1) and this is depicted in Fig. 4(a) 
in the plot of $<\ln \left(Z_{\max }\right)>$ vs $\ln \left|\left(n-n_{c}\right)\right|$. The value of $\beta$ is $0.241 \pm 0.011$. Campi [3] has pointed out that one can determine the value of the exponent $\tau$ from the slope of $\left\langle\ln S_{3}>\right.$ vs $\ln S_{2}$, where we use the gas branch of the plot and obtain a value of $\tau_{g}=2.038 \pm 0.056$ and this is shown in Fig. 4(b). $S_{2}$ and $S_{3}$ assume their maximum around the critical value of $n_{c}$. Above the critical zone, points fall closer to the origin representing fragments which are generally produced with relatively small impact parameters. The $\tau$ value obtained from Fig. $1(a)$ is slightly higher than that obtained from Fig. 4(b). Generally, on a relatively small lattice, it is rather hard to determine $\tau$ values very accurately. But overall, the values of $\beta, \gamma$ and $\tau$ determined by using the above method obey the scaling relation given by $\tau=2+\beta /(\beta+\gamma)$.

The values of critical exponents $\gamma, \beta$ and $\tau$ are listed in Table I along with those at low energy beam [4] and at higher energy beam of ${ }^{207} \mathrm{~Pb}$ at $160 \mathrm{~A}$ $\mathrm{GeV}$. These values at different energies (higher and lower) are quite similar within their statistical errors. The small difference may be due to the fact that at lower energy [4] they may have used the cascade protons which might have been produced from the projectile or from the target. But we have used only the projectile (spectator) protons. Exponents from these two experiments are further compared with three-dimensional systems possessing a scalar order parameter, liquid-gas [1], percolation [1] and mean field limit of liquid-gas system [11] which are also listed in Table 1. We find that our values are close to the point for liquid-gas systems and are significantly away from either the percolation or liquid-gas mean field results. Critical exponents describe the powerlaw behavior of thermodynamical quantities as the critical point is approached.

We thus conclude that despite the large differences in beam energies, detection technique, different critical multiplicity $\left(n_{c}\right)$ cuts and different targets; nearly identical exponents values are observed for liquid-gas system at different energies. We think that the technique used here is quite valid as the moments of charged nuclear fragmentation from ${ }^{197} \mathrm{Au}$ beam at $10.6 \mathrm{~A} \mathrm{GeV}$ provide evidence for critical behavior in finite nuclei. The values of the critical exponents are very close to those expected in a liquidgas phase transition and this indicates that a phase transition has taken place at $10.6 \mathrm{~A} \mathrm{GeV}$. The technique used for the extraction of critical exponents is reasonably valid and should be tested with other nuclear systems. This will help to establish the values of critical exponents of nuclear matter.

We are thankful to the BNL technical staff for the exposure and to Prof. G. Romano for the development of the emulsions. This research work was partially supported by the D.O.E. and the SUNY Research Foundation at Buffalo.

\section{References}

[1] D. Stauffer, Phys. Rep. 54, 1 (1979); D. Stauffer and A. Aharony, Introduction to Percolation Theory (Taylor and Francis, London, 1992) 2nd. ed; H. E. Stanley, Introduction to Phase Transitions and Critical Phenomena (Oxford University Press, Oxford, 1971).

[2] J. E. Finn et al., Phys. Rev. Lett. 49, 1321 (1982); R. W. Munich et al., Phys. Lett. B 118,458 (1982).

[3] X. Campi, J. Phys. A18 L917 (1986) and Phys. Lett. B 208, 351 (1988).

[4] M. L. Gilkes et al., Phys. Rev. Lett. 73, 1590 (1994); J. B. Elliot et al., Phys. Rev. C 49 3185 (1994) and the references therein.

[5] P. L. Jain and G. Singh, Phys. Lett. B 382, 289 (1996)

[6] P. L. Jain, G. Singh and A. Mukhopadhyay, Phys. Rev. C 50, 1085 (1994).

[7] P. L. Jain and G. Singh, Nucl. Phys. A 591, 711 (1995).

[8] J. Hubele et al., Phys. Rev. C 46, R1577 (1992).

[9] P. L. Jain, G. Singh and A. Mukhopadhyay, Phys. Rev. Lett. 74, 1534 (1995).

[10] L. G. Moretto et al., Phys. Rev. Lett. 74, 1530 (1995).

[11] H. B. Callen, Thermodynamics and Introduction to Thermostatistics (John Wiley and Sons, New York 1985, 2nd ed.)

Figure Captions

Fig. 1 (a) Relationship between $\ln N_{Z}$ and $\ln Z$ for $2 \leq Z \leq 23$. Solid line is a power-law fit, $Z^{-T}$. (b) $\ln N_{Z}$ vs $Z$ for $3 \leq Z \leq 11$. (c) Normalized multiplicity distribution of PFs with 
$3 \leq Z \leq 11$ in events with "cold" (closed circles) and "hot" (open circles) alphas. (d) Multiplicity distribution of PFs with $3 \leq Z \leq 11$ in events with: (i) $N_{p} \leq 28$ (closed circles) and (ii) $28<N_{p} \leq 60$ (open circles). Dotted lines in Figs. 1(b), 1(c) and 1(d) are the exponential fits, $e^{-\alpha Z}$, through the data points.

Fig. 2 (a) Distribution of $\ln N$ as a function of $E_{t}^{-1 / 2}$. Solid line is an exponential fit to the data (see text). (b) Correlation of $\left\langle E_{t}^{1 / 2}\right\rangle$ vs $Z_{\text {bound }}$. Solid line is a linear fit to the data points. (c) Variation of $\left\langle\ln S_{2}>\right.$ with $n$ for PFs with $Z \geq 1$. (d) Energy density $\epsilon_{0}$ as a function of $N_{s}$ for shower particles in the range $0.5 \leq \eta \leq 3.7$. Dotted line is a linear fit to the data points.

Fig. 3 (a) A plot of $\left|\gamma_{q}-\gamma_{l}\right|$ vs $n_{c}$. The variation of $\left\langle\ln S_{2}>\right.$ as a function of $\ln \left|\left(n-n_{n}\right)\right|$ for the ${ }^{197} \mathrm{Au}$ events in: (b) liquid zone and (c) gas zone. The solid lines correspond to the best fits.

Fig. 4 For ${ }^{197} \mathrm{Au}$ data: (a) $<\ln \left(Z_{\max }\right)>$ as a function of $\ln \left|\left(n-n_{c}\right)\right|$ in the liquid zone and (b) $<\ln S_{3}>$ as a function of $\ln S_{2}$ in gas zone. The solid lines are the best fits to the data points. Errors shown in Fig. 4(a) are the standard deviations from the mean value.
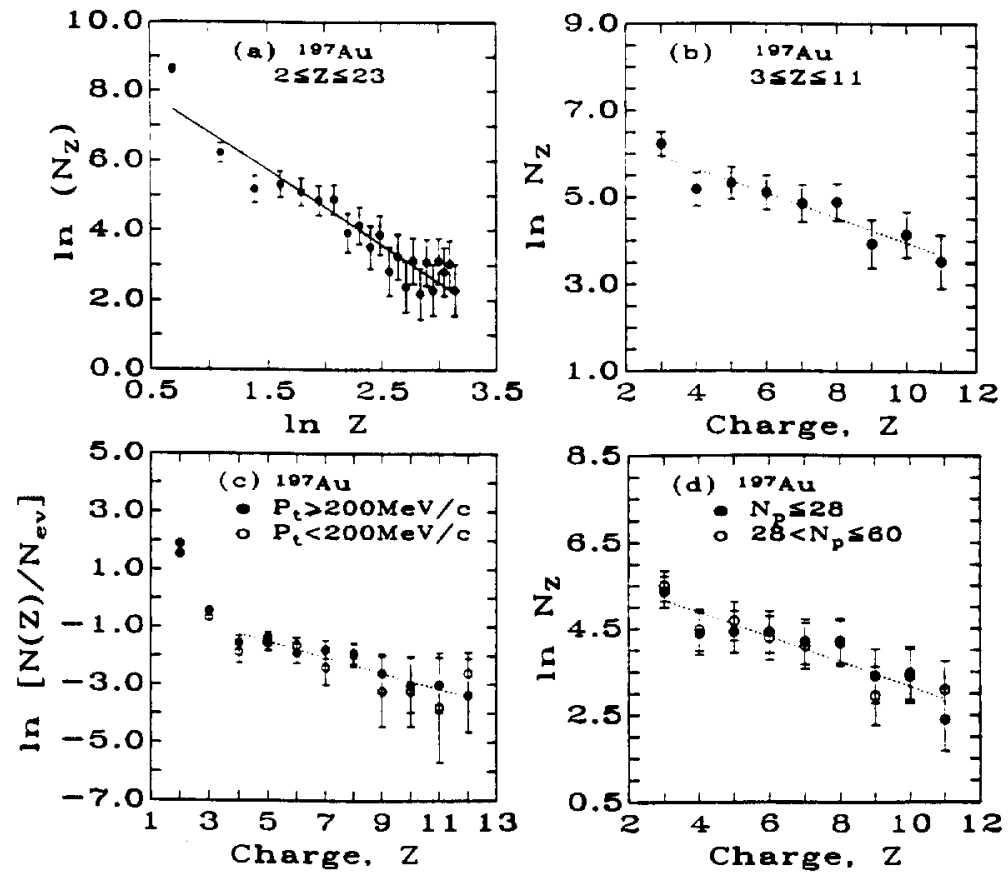

Jain et al. Fig. 1
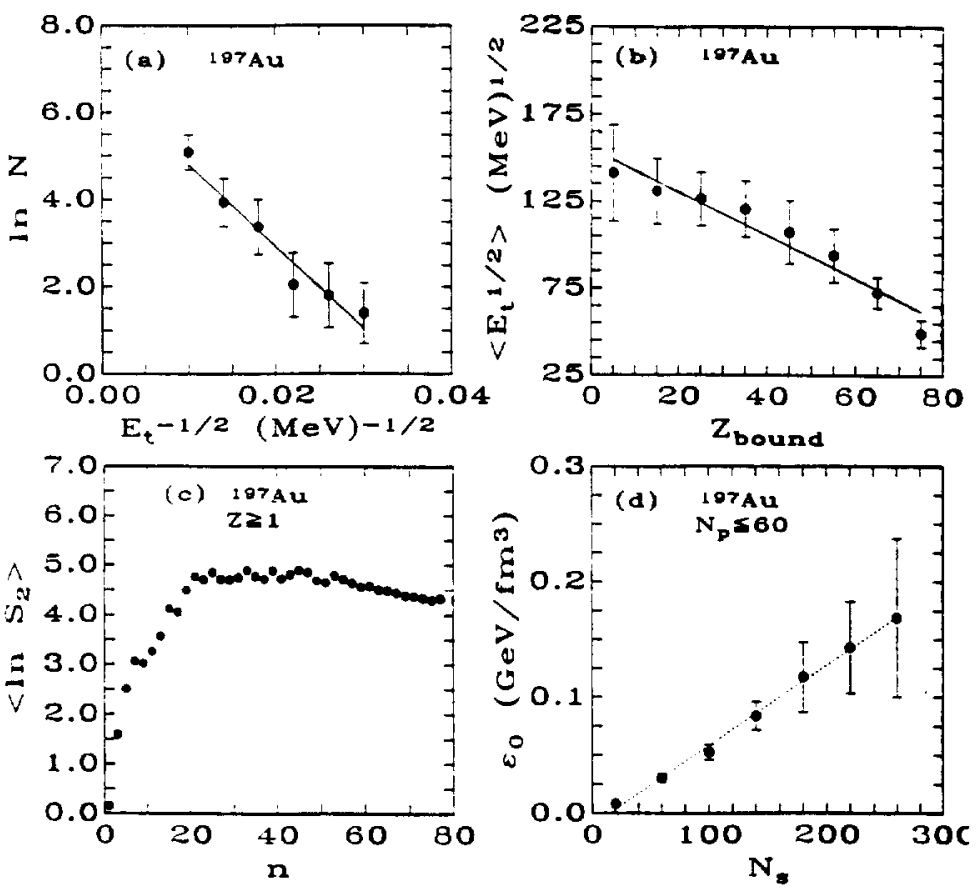

Jain et al. Fig. 2
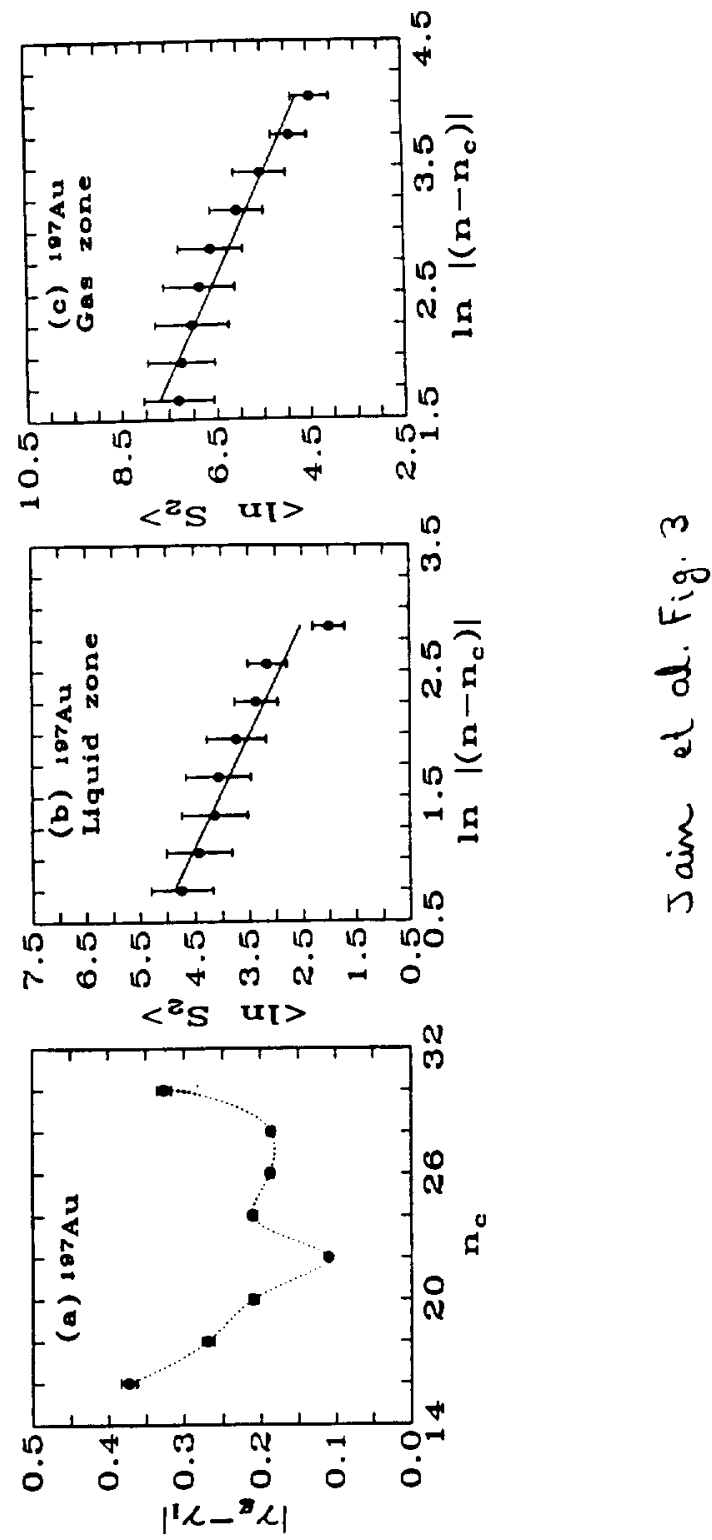

3 
Table I. Critical multiplicity and exponents for the ${ }^{197} \mathrm{Au}$ and ${ }^{207} \mathrm{~Pb}$ projectile fragments and other three dimensional systems.

\begin{tabular}{ccccccc}
\hline \hline Parameter & This Expt. & Ref. [5] & Ref. [4] & Liquid-gas & Percolation & $\begin{array}{c}\text { Liquid-gas } \\
\text { mean field }\end{array}$ \\
\hline$n_{c}$ & $22 \pm 1$ & $38 \pm 1$ & $26 \pm 1$ & - & - & \\
$\gamma$ & $1.17 \pm 0.19$ & $1.066 \pm 0.082$ & $1.4 \pm 0.1$ & 1.23 & 1.8 & 1.0 \\
$\beta$ & $0.241 \pm 0.011$ & $0.264 \pm 0.018$ & $0.29 \pm 0.02$ & 0.33 & 0.41 & 0.5 \\
$\tau$ & $2.038 \pm 0.056$ & $2.17 \pm 0.15$ & $2.14 \pm 0.06$ & 2.21 & 2.18 & 2.33 \\
\hline \hline
\end{tabular}
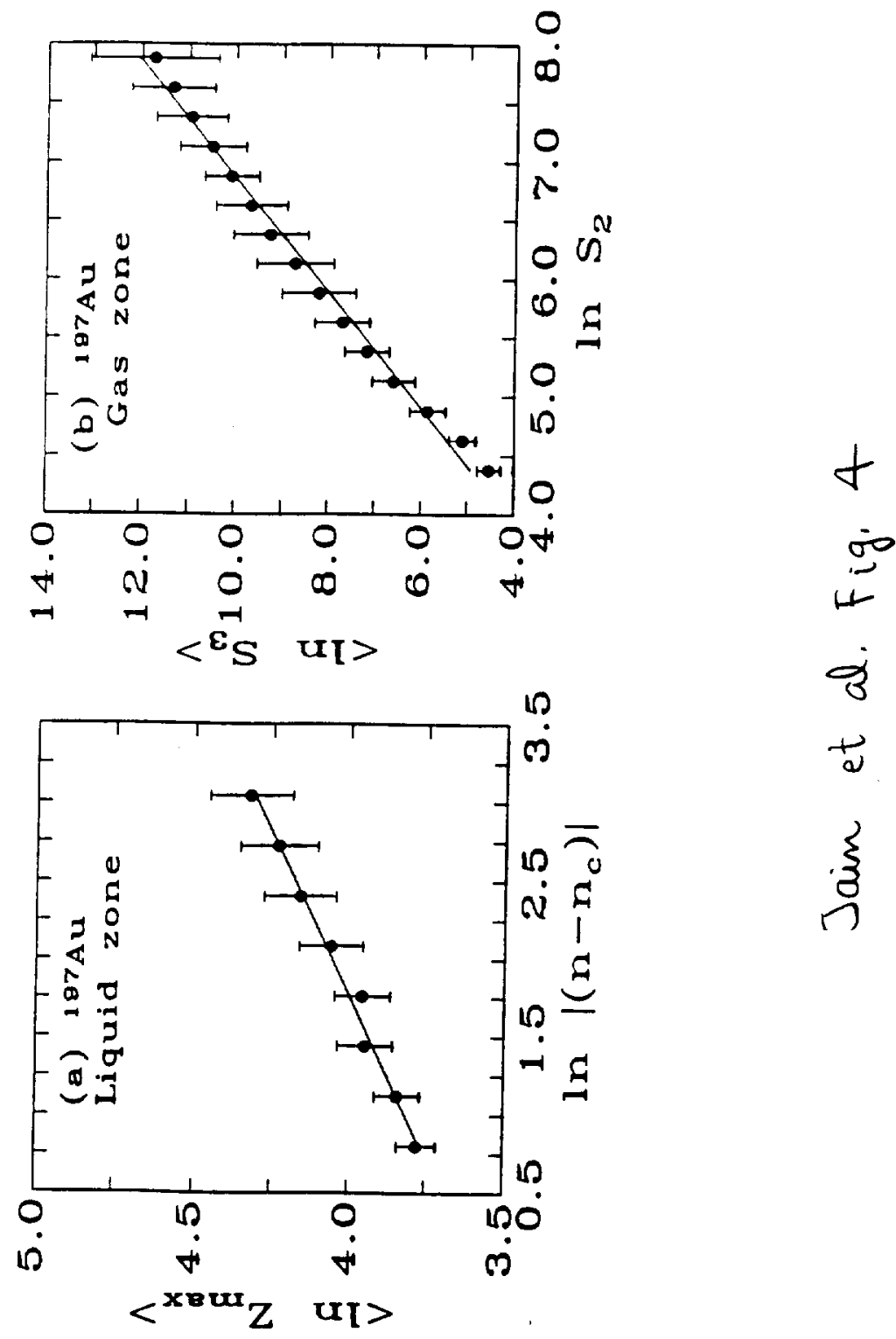

$+$

! 